\title{
ON THE ABSOLUTE NÖRLUND SUMMABILITY FACTORS OF INFINITE SERIES
}

\author{
S. R. SINGH
}

ABstract. Taking the start from an earlier result on the absolute harmonic summability factors due to $\mathrm{S}$. N. Lal, we obtain in this paper suitable factors $\left\{\epsilon_{n}\right\}$ so that the series $\sum a_{n} \epsilon_{n}$ may be summable $\left|N, p_{n}\right|$, whenever the series $\sum a_{n}$ is summable $|C, 1|$.

1. Let $\left\{S_{n}\right\}$ denote the $n$th partial sum of the series $\sum a_{n}$. Let $\left\{p_{n}\right\}$ be a sequence of real numbers and let us write

$$
P_{n}=p_{0}+p_{1}+p_{2}+\cdots+p_{n}, \quad P_{-1}=p_{-1}=0 .
$$

The sequence to sequence transformation,

$$
t_{n}=\frac{1}{P_{n}} \sum_{\nu=0}^{n} p_{n-\nu} S_{\nu}=\frac{1}{P_{n}} \sum_{\nu=0}^{n} P_{n-\nu} a_{\nu} \quad\left(P_{n} \neq 0\right),
$$

defines the sequence $\left\{t_{n}\right\}$ of Nörlund means of the sequence $\left\{S_{n}\right\}$, generated by the sequence of coefficients $\left\{p_{n}\right\}$.

The series $\sum a_{n}$ is said to be absolutely summable $\left(N, p_{n}\right)$, or summable $\left|N, p_{n}\right|$, if the sequence $\left\{t_{n}\right\}$ is of bounded variation [6], that is,

$$
\sum_{n}\left|t_{n}-t_{n-1}\right|<\infty \text {. }
$$

In the special case in which $p_{n}=1 /(n+1)$, the summability $\left|N, p_{n}\right|$ is the same as absolute harmonic summability.

Also, when

$$
p_{n}=\left(\begin{array}{c}
n+\alpha-1 \\
\alpha-1
\end{array}\right)=\frac{\Gamma(n+\alpha)}{\Gamma(n+1) \Gamma(\alpha)} \quad(\alpha \geqq 0),
$$

the Nörlund mean reduces to the familiar $(C, \alpha)$ mean. Thus the summability $\left|N, p_{n}\right|$, when $p_{n}$ is given by (1.2) is the same as the summability $|C, \alpha|$.

2. Throughout the present paper we write

$$
B_{n}=\sum_{\nu=1}^{n} \nu a_{\nu}, \quad \Delta \chi_{n}=\chi_{n}-\chi_{n+1},
$$

Received by the editors November 13, 1969.

AMS Subject Classifications. Primary 4010, 4030, 4031, 4032; Secondary 4220.

Key Words and Phrases. Nörlund means, absolutely summable $\left(N, p_{n}\right)$, absolute harmonic summability, summability $|C, \alpha|$, convex sequences, absolute summability factor theorem. 
and $C$ to denote an absolute constant not necessarily the same at each occurrence.

3. In a paper in this journal Lal [4] established the following

THEOREM A. If $B_{n}=O(n)$, then the series $\sum a_{n} \log (n+1) \lambda n / n$, where $\left\{\lambda_{n}\right\}$ is a convex sequence, such that $\sum n^{-1} \lambda_{n}$ is convergent, is absolutely harmonic summable.

With the help of this theorem Lal obtained a result on absolute harmonic summability factors of Fourier series (see [4]). These results were extended by several workers such as Bhatt [2], Mohapatra, Das and Srivastava [8], Mehrotra [7], Ahmad [1], and others.

In this paper we establish the following absolute summability factor theorem, which when combined with a known result yields a result more general than Theorem A (see $\S 6$, Theorem 2).

Theorem 1. Let $p_{0}>0$ and $p_{n}$ be a nonnegative and nonincreasing sequence. If a series $\sum a_{n}$ is summable $|C, 1|$, and a sequence $\left\{\epsilon_{n}\right\}$ is such that,

$$
n \epsilon_{n}=O\left(P_{n}\right)
$$

and

$$
n \Delta \epsilon_{n}=O(1),
$$

as $n \rightarrow \infty$, then the series $\sum a_{n} \epsilon_{n}$ is summable $\left|N, p_{n}\right|$.

It is interesting to note that the following form of a result due to Kogbetliantz ${ }^{1}$ [3] follows as a corollary of our theorem.

Corollary. If a series $\sum a_{n}$ is summable $|C, 1|$, then the series $\sum a_{n} \epsilon_{n}$ is summable $|C, \beta|(0<\beta \leqq 1)$,

where

$$
\epsilon_{n}=1 /(n+1)^{1-\beta} .
$$

4. We require the following lemma to establish our theorem.

LEMмA [1]. If $p_{0}>0$ and $p_{n}$ is a nonnegative and nonincreasing sequence, then for $\nu \geqq 1$

$$
\begin{array}{r}
\sum_{n=\nu}^{\infty} \frac{p_{n} p_{n-\nu}}{P_{n} P_{n-1}}-C \\
\sum_{n=\nu}^{\infty} \frac{p_{n}\left(P_{n}-P_{n-\nu}\right)}{P_{n} P_{n-1}} \leqq C,
\end{array}
$$

${ }^{1}$ In 1952 Peyerimhoff [9], gave a simpler proof of Kogbetliantz's result. 


$$
\sum_{n=\nu}^{\infty} \frac{\left|\Delta_{n} p_{n-\nu-1}\right|}{P_{n-1}} \leqq \frac{C}{P_{\nu}}+\frac{C}{\nu},
$$

and

$$
\sum_{n=\nu}^{\infty} \frac{\left(p_{n-\nu}-p_{n}\right)}{P_{n-1}} \leqq C .
$$

5. Proof of the theorem. Let $\tau_{n}$ denote the $n$th Nörlund mean of the series $\sum a_{n} \epsilon_{n}$. Then by definition,

$$
\tau_{n}=\frac{1}{P_{n}} \sum_{\nu=0}^{n} p_{n-\nu} \sum_{\mu=0}^{\nu} a_{\mu} \epsilon_{\mu}=\frac{1}{P_{n}} \sum_{\nu=0}^{n} P_{n-\nu} a_{\nu} \epsilon_{\nu},
$$

so that

$$
\begin{aligned}
\tau_{n}-\tau_{n-1}= & \frac{1}{P_{n} P_{n-1}} \sum_{\nu=1}^{n}\left(P_{n} p_{n-\nu}-P_{n-\nu} p_{n}\right) \epsilon_{\nu} a_{\nu} \\
= & \frac{p_{n}}{P_{n} P_{n-1}} \sum_{\nu=1}^{n}\left(P_{n}-P_{n-\nu}\right) \epsilon_{\nu} a_{\nu} \\
& +\frac{1}{P_{n-1}} \sum_{\nu-1}^{n}\left(p_{n-\nu}-p_{n}\right) \epsilon_{\nu} a_{\nu} \\
= & \frac{p_{n}}{P_{n} P_{n-1}} \sum_{\nu=1}^{n-1} \Delta_{\nu}\left\{\left(P_{n}-P_{n-\nu}\right) \frac{\epsilon_{\nu}}{\nu}\right\} B_{\nu} \\
& +\frac{p_{n}}{P_{n} P_{n-1}}\left\{\left(P_{n}-P_{0}\right) \frac{\epsilon_{n}}{n}\right\} B_{n} \\
& +\frac{1}{P_{n-1}} \sum_{\nu=1}^{n-1} \Delta_{\nu}\left\{\left(p_{n-\nu}-p_{n}\right) \frac{\epsilon_{\nu}}{\nu}\right\} B_{\nu} \\
& -\frac{1}{P_{n-1}}\left\{\left(p_{0}-p_{n}\right) \frac{\epsilon_{n}}{n}\right\} B_{n} \\
= & \frac{p_{n}}{P_{n} P_{n-1}} \sum_{\nu=1}^{n} \Delta_{\nu}\left\{\left(P_{n}-P_{n-\nu}\right) \frac{\epsilon_{\nu}}{\nu}\right\} B_{\nu} \\
& +\frac{1}{P_{n-1}} \sum_{\nu=1}^{n} \Delta_{\nu}\left\{\left(p_{n-\nu}-p_{n}\right) \frac{\epsilon_{\nu}}{\nu}\right\} B_{\nu},
\end{aligned}
$$

and therefore 
1970]

$$
\begin{aligned}
\left|\tau_{n}-\tau_{n-1}\right| \leqq & \frac{p_{n}}{P_{n} P_{n-1}} \sum_{\nu=1}^{n}\left|\Delta_{\nu}\left\{\left(P_{n}-P_{n-\nu}\right) \frac{\epsilon_{\nu}}{\nu}\right\}\right|\left|B_{\nu}\right| \\
& +\frac{1}{P_{n-1}} \sum_{\nu=1}^{n}\left|\Delta_{\nu}\left\{\left(p_{n-\nu}-p_{n}\right) \frac{\epsilon_{\nu}}{\nu}\right\}\right|\left|B_{\nu}\right| .
\end{aligned}
$$

Thus to establish the theorem we have to show that

$$
\Sigma_{1}=\sum_{n=1}^{\infty} \frac{p_{n}}{P_{n} P_{n-1}} \sum_{\nu=1}^{n}\left|\Delta_{\nu}\left\{\left(P_{n}-P_{n-\nu}\right) \frac{\epsilon_{\nu}}{\nu}\right\}\right|\left|B_{\nu}\right|<\infty,
$$

and

$$
\Sigma_{2}=\sum_{n=1}^{\infty} \frac{1}{P_{n-1}} \sum_{\nu=1}^{n}\left|\Delta_{\nu}\left\{\left(p_{n-\nu}-p_{n}\right) \frac{\epsilon_{\nu}}{\nu}\right\}\right|\left|B_{\nu}\right|<\infty .
$$

Before proceeding to the proof of (5.1) and (5.2), we observe that, under the hypothesis (3.1) of our theorem $\epsilon_{n}=O\left(P_{n} / n\right)=O(1)$, as $n \rightarrow \infty$ since $P_{n} / n$ is monotonic nonincreasing [5]. We now have

$$
\begin{aligned}
\Sigma_{1}= & O(1) \sum_{n=1}^{\infty} \frac{p_{n}}{P_{n} P_{n-1}} \sum_{\nu=1}^{n}\left(P_{n}-P_{n-\nu}\right)\left|\epsilon_{\nu}\right| \frac{\left|B_{\nu}\right|}{\nu^{2}} \\
& +O(1) \sum_{n=1}^{\infty} \frac{p_{n}}{P_{n} P_{n-1}} \sum_{\nu=1}^{n}\left(P_{n}-P_{n-\nu}\right)\left|\Delta \epsilon_{\nu}\right| \frac{\left|B_{\nu}\right|}{(\nu+1)} \\
& +O(1) \sum_{n=1}^{\infty} \frac{p_{n}}{P_{n} P_{n-1}} \sum_{\nu=1}^{n} p_{n-\nu}\left|\epsilon_{\nu+1}\right| \frac{\left|B_{\nu}\right|}{(\nu+1)} \\
& +O(1) \sum_{\nu=1}^{\infty} \frac{\left|\epsilon_{\nu}\right|\left|B_{\nu}\right|}{\nu^{2}} \sum_{n=\nu}^{\infty} \frac{p_{n}\left(P_{n}-P_{n-\nu}\right)}{P_{n} P_{n-1}} \\
& +O(1) \sum_{\nu=1}^{\infty} \frac{\left|\Delta \epsilon_{\nu}\right|\left|B_{\nu}\right|}{(\nu+1)} \sum_{n=\nu}^{\infty} \frac{p_{n}\left(P_{n}-P_{n-\nu}\right)}{P_{n} P_{n-1}} \\
& +O(1) \sum_{\nu=1}^{\infty} \frac{\left|\epsilon_{\nu+1}\right|\left|B_{\nu}\right|}{(\nu+1)} \sum_{n=\nu}^{\infty} \frac{p_{n} p_{n-\nu}}{P_{n} P_{n-1}} \\
= & O(1) \sum_{\nu=1}^{\infty} \frac{\left|\epsilon_{\nu}\right|\left|B_{\nu}\right|}{\nu^{2}}+O(1) \sum_{\nu=1}^{\infty} \frac{\left|\Delta \epsilon_{\nu}\right|\left|B_{\nu}\right|}{\nu} \\
= & O(1) \sum_{\nu=1}^{\infty} \frac{\left|\epsilon_{\nu}\right|\left|B_{\nu}\right|}{\nu^{2}}+O(1) \sum_{\nu=1}^{\infty} \frac{\nu\left|\Delta \epsilon_{\nu}\right|\left|B_{\nu}\right|}{\nu^{2}} \\
= & O(1) \sum_{\nu=1}^{\infty} \frac{\left|B_{\nu}\right|}{\nu^{2}} \\
= & O(1)
\end{aligned}
$$


by (4.1), (4.2) of the lemma and under the hypotheses of the theorem (since the series $\sum a_{n}$ is summable $\left.|C, 1|, \sum\left|B_{n}\right| / n^{2}<\infty\right)$ ). Again,

$$
\begin{array}{rl}
\Sigma_{2}= & O(1) \sum_{n=1}^{\infty} \frac{1}{P_{n-1}} \sum_{\nu=1}^{n}\left(p_{n-\nu}-p_{n}\right)\left|\epsilon_{\nu}\right| \frac{\left|B_{\nu}\right|}{\nu^{2}} \\
& +O(1) \sum_{n=1}^{\infty} \frac{1}{P_{n-1}} \sum_{\nu=1}^{n}\left(p_{n-\nu}-p_{n}\right)\left|\Delta \epsilon_{\nu}\right| \frac{\left|B_{\nu}\right|}{(\nu+1)} \\
& +O(1) \sum_{n=1}^{\infty} \frac{1}{P_{n-1}} \sum_{\nu=1}^{n}\left|\Delta_{n} p_{n-\nu-1}\right|\left|\epsilon_{\nu+1}\right| \frac{\left|B_{\nu}\right|}{(\nu+1)} \\
= & O(1) \sum_{\nu=1}^{\infty} \frac{\left|\epsilon_{\nu}\right|\left|B_{\nu}\right|}{\nu^{2}} \sum_{n=\nu}^{\infty} \frac{\left(p_{n-\nu}-p_{n}\right)}{P_{n-1}} \\
& +O(1) \sum_{\nu=1}^{\infty} \frac{\left|\Delta \epsilon_{\nu}\right|\left|B_{\nu}\right|}{(\nu+1)} \sum_{n=\nu}^{\infty} \frac{\left(p_{n-\nu}-p_{n}\right)}{P_{n-1}} \\
& +O(1) \sum_{\nu=1}^{\infty} \frac{\left|\epsilon_{\nu+1}\right|\left|B_{\nu}\right|}{(\nu+1)} \sum_{n=\nu}^{\infty} \frac{\left|\Delta_{n} p_{n-\nu-1}\right|}{P_{n-1}} \\
= & O(1) \sum_{\nu=1}^{\infty} \frac{\left|\epsilon_{\nu}\right|\left|B_{\nu}\right|}{\nu^{2}}+O(1) \sum_{\nu=1}^{\infty} \frac{\left|\Delta \epsilon_{\nu}\right|\left|B_{\nu}\right|}{\nu} \\
& +O(1) \sum_{\nu=1}^{\infty} \frac{\left|\epsilon_{\nu+1}\right|\left|B_{\nu}\right|}{(\nu+1) P_{\nu}}+O(1) \sum_{\nu=1}^{\infty} \frac{\left|\epsilon_{\nu+1}\right|\left|B_{\nu}\right|}{\nu(\nu+1)} \\
= & O(1) \sum_{\nu=1}^{\infty} \frac{\left|\epsilon_{\nu}\right|\left|B_{\nu}\right|}{\nu^{2}}+O(1) \sum_{\nu=1}^{\infty} \frac{\nu\left|\Delta \epsilon_{\nu}\right|\left|B_{\nu}\right|}{\nu^{2}} \\
& +O(1) \sum_{\nu=1}^{\infty} \frac{\left|B_{\nu}\right|}{\nu(\nu+1) \epsilon_{\nu+1}|| B_{\nu} \mid} \\
\nu^{2} & O(1)
\end{array}
$$

by (4.3), (4.4) of the lemma and under the hypotheses of the theorem.

Combining the estimates of $\Sigma_{1}$ and $\Sigma_{2}$, we see that the theorem is completely established.

6. Prasad and Bhatt [10] established the following

THEOREM B. If $\left\{\lambda_{n}\right\}$ is a convex sequence, such that $\sum n^{-1} \lambda_{n}$ is convergent, and 


$$
\frac{1}{n} \sum_{\nu=1}^{n} \frac{\left|B_{\nu}\right|}{\nu}=O(\log n)^{k} \quad(k \geqq 0),
$$

then the series $\sum\{\log (n+1)\}^{-k} \lambda_{n} a_{n}$ is summable $|C, 1|$.

If we combine the above result with Theorem 1 we get the following

Theorem 2. If $\left\{\lambda_{n}\right\}$ is a convex sequence, such that $\sum n^{-1} \lambda_{n}$ is convergent, and if (6.1) holds then the series $\sum\left(a_{n} \lambda_{n} \epsilon_{n} /\{\log (n+1)\}^{k}\right)$ is summable $\left|N, p_{n}\right|$, provided that

$$
n \epsilon_{n}=O\left(P_{n}\right), \text { and } n \Delta \epsilon_{n}=O(1),
$$

as $n \rightarrow \infty$.

Finally, the author expresses his warmest thanks to Dr. S. N. Lal for his valuable guidance and helpful suggestions. Thanks are also due to the University Grants Commission for the financial assistance.

\section{REFERENCES}

1. Z. U. Ahmad, On the absolute Nörlund summability factors of a Fourier series, Riv. Mat. Univ. Parma (2) 7 (1966), 157-169. MR 37 \#6674.

2. S. N. Bhatt, On the absolute summability of factored Fourier series, Ann. Mat. Pura Appl. (4) 72 (1966), 253-265. MR 35 \#657.

3. E. Kogbetliantz, Sur les séries absolument sommables par la méthode des moyennes arithmetiqués, Bull. Sci. Math. (2) 49 (1925), 234-256.

4. S. N. Lal, On the absolute harmonic summability of the factored Fourier series, Proc. Amer. Math. Soc. 14 (1963), 311-319. MR 26 \#5344.

5. L. McFadden, Absolute Nörlund summability, Duke Math. J. 9 (1942), 168207. MR 3, 295.

6. F. M. Mears, Some multiplication theorems for Nörlund means, Bull. Amer. Math. Soc. 41 (1935), 875-880.

7. N. D. Mehrotra, On the absolute Nörlund summability of a factored Fourier Series, Proc. Japan Acad. 41 (1965), 46-51. MR 31 \#5037.

8. R. N. Mohapatra, G. Das and V. P. Srivastava, On absolute summability factors of infinite series and their application to Fourier series, Proc. Cambridges Philos. Soc. 63 (1967), 107-118. MR 34 \#3155.

9. A. Peyerimhoff, Über einen satz von Hernn Kogbetliantz aus der Theorie der absoluten Cesdroschen Summierbarkeit, Arch. Math., 3 (1952), 262-265. MR 14, 551.

10. B. N. Prasad and S. N. Bhatt, The summability factors of a Fourier series, Duke Math. J. 24 (1957), 103-117. MR 18, 892.

Banaras Hindu University, Varanasi-5, India 